\title{
Development of TEM-SXES instruments for valence electron spectroscopy
}

\author{
M. Terauchi
}

Institute of Multidisciplinary Research for Advanced Materials, Tohoku University, 2-1-1 Katahira, Aoba-ku, Sendai 980-8577, Japan.

Characterization of the electronic states of valence electrons (bonding electrons) is imperative for characterizing new functional materials. Furthermore, it is expected with high special resolution and based on microscopy. For that purpose, soft-X-ray emission spectroscopy (SXES), which is caused especially by transitions of valence electrons to inner-shell core-hole states, combined with transmission electron microscopy should be the best way. We have been developing SXES instruments for a conventional analytical transmission electron microscope (TEM) [1-3]. Those spectrometers are a wavelength-dispersive type but do not disturb the spatial resolution of a TEM, because those do not have a moving mechanism as in the case of wavelength-dispersive spectrometers of EPMA. Each spectrometer was composed of laminar-type varied-line-spaced (VLS) gratings, a back-illumination CCD detector without anti-reflection coating and X-ray collection mirrors.

Figure 1 shows carbon K-emission spectra of single crystals of diamond, graphite and monomer $\mathrm{C}_{60}$. Crystal structures of those materials are also shown. Those spectra were obtained by using a grating of a groove density of $1600 \mathrm{line} / \mathrm{mm}$ with $\mathrm{Ni}$ coating for improving reflection efficiency. The energy dispersion for carbon K-emission of $280 \mathrm{eV}$ is $0.13 \mathrm{eV} / \mathrm{CCD}$ pixel. Dominant peak positions indicated by vertical lines, assigned to $\sigma$-bonding states, are different for diamond and graphite due to different $\sigma$-bond lengths for the materials. The spectrum of monomer $\mathrm{C}_{60}$ crystal, which is formed by weakly bonded fullerene molecules (curved graphene sheet), shows the $\sigma$-bonding states almost at the same energy with that of graphite. Shoulder structures of the $\mathrm{C}_{60}$ spectrum indicated by vertical arrows correspond to $\pi$-bonding states. Those are assigned to $h_{u}$ (right hand side) and $h_{g}+g_{g}$ (left hand side) states of the band diagram of $\mathrm{C}_{60}$ monomer crystal [4]. It is seen a characteristic gap between the $\pi$-bonding and the $\sigma$-bonding states.

When a material has anisotropic bonding, SXES emission due to transitions of the anisotropic bonding electrons to inner core-hole states shows an anisotropic intensity distribution. The upper figure of Fig. 2 shows carbon K-emission spectra obtained at 60 deg. and 15 deg. take-off angles, which are angles between the emission (detection) direction and (0001) plane of graphite. It is clearly seen that the two spectra show a different intensity distribution. Emission intensities from $\pi$-bonding and $\sigma$-bonding states depend on the take-off angle $\theta$ as $\cos ^{2} \theta$ and $1+\sin ^{2} \theta$, respectively. Thus, $\pi$-bonding and $\sigma$-bonding states can be derived from the two spectra as shown in the lower part of Fig.2. The lateral axis numbers are the pixel number of the detector. Band widths of $\sigma$ - and $\pi$-bands and the relative energy position are consistent with a theoretical calculation. It should be noted that the gap appeared in the monomer $\mathrm{C}_{60}$ crystal between the $\pi$-band and the $\sigma$-band are overlapped in graphite. This overlap is seen around the $\Gamma$ point in the band diagram. This experimental result gives a nice reference for critical researches of carbon materials based on grapheme network. 
References

[1] M.Terauchi, H.Yamamoto and M.Tanaka, J. Electron Microscopy, 50(2) (2001) 101.

[2] M.Terauchi and M.Kawana, Ultramicroscopy, 106 (2006) 1069.

[3] M.Terauchi et al., Microsc. Microanal., 12 suppl.2 (2006) 866.

[4] S.Saito and A.Oshiyama, Phys. Rev. Lett., 66 (1991) 2637.

Fig.1 Carbon K-emission spectra of single crystals of diamond, graphite and monomer $\quad \mathrm{C}_{60}$. Crystal structures of those materials are also shown. Shoulder structures of the $\mathrm{C}_{60}$ spectrum indicated by vertical arrows correspond to $\pi$-bonding states of $h_{u}$ (right hand side) and $h_{g}+g_{g}$ states.

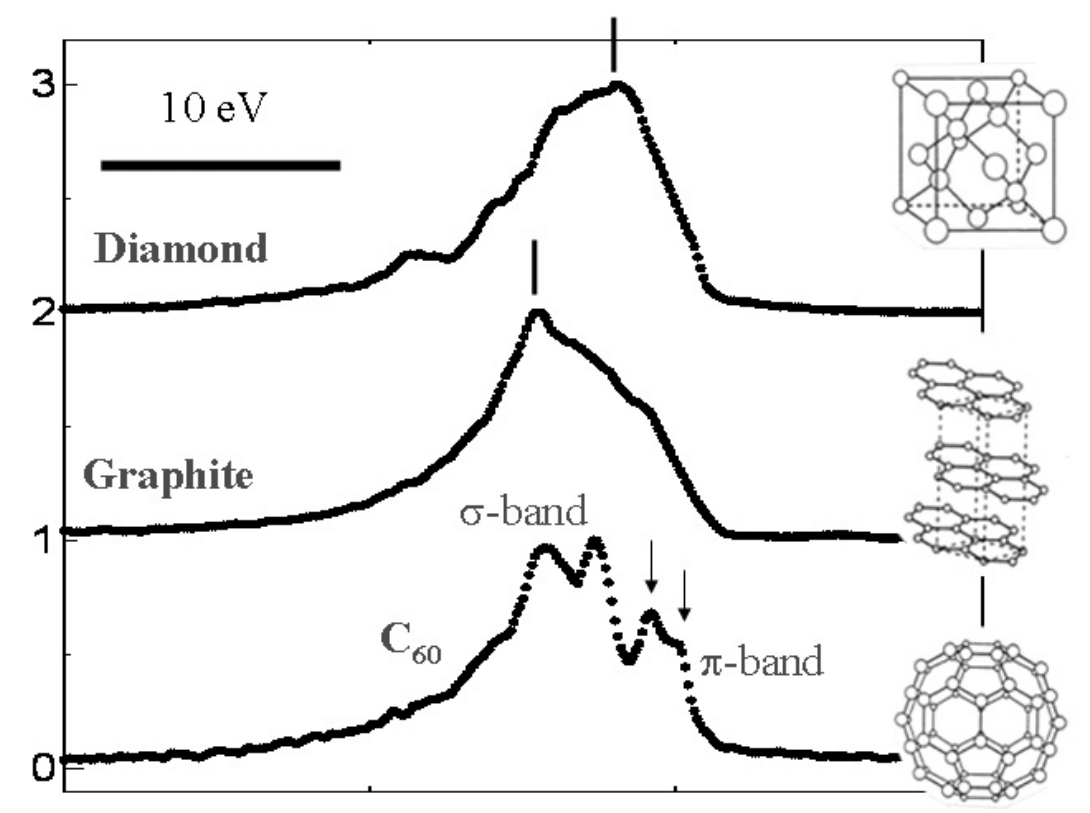

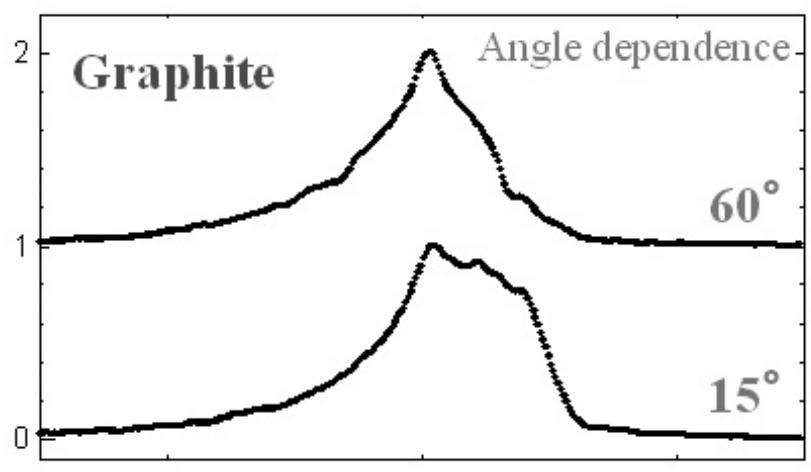

Derivation

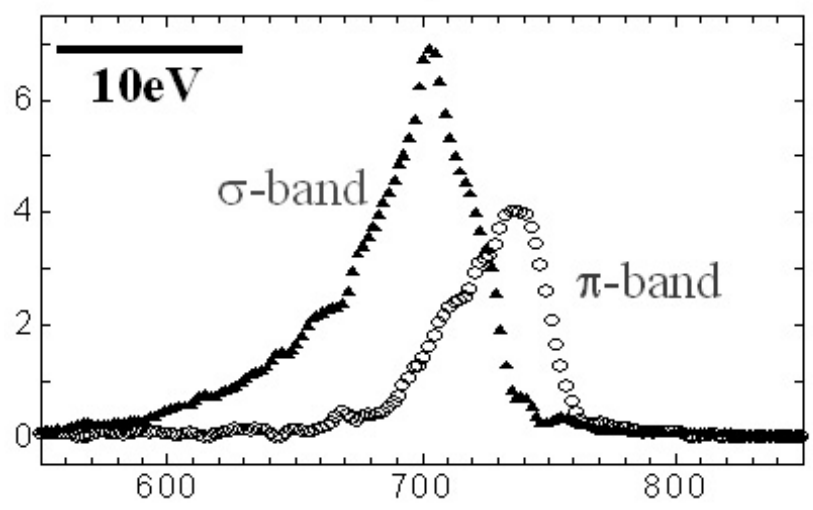

Fig.2 Upper part shows carbon K-emission spectra obtained at $60 \mathrm{deg}$. and $15 \mathrm{deg}$. take-off angles. The lower part shows numerically derived $\pi$-bonding and $\sigma$-bonding state distributions from the two spectra. The overlap of $\pi$ - and $\sigma$-bands corresponds to around the $\Gamma$ point in the band diagram. 